M+A Revista Electrónica de Medioambiente

ISSN-e: $1886-3329$

http://dx.doi.org/10.5209/MARE.58370

\title{
Efecto de técnicas de agricultura de conservación en la respiración del suelo en plantaciones de almendro en la región mediterránea (SE España)
}

\author{
Carmen Rocío Pleguezuelo'; J.R. Francia Martínez1; Belén Cárceles Rodríguez²; \\ B. Gálvez Ruiz1; I.F. García Tejero; Victor Hugo Durán Zuazo ${ }^{3}$
}

Recibido: 21 de agosto del 2017/ Enviado a evaluar: 19 de septiembre del 2017/ Aceptado: 10 de noviembre del 2017

Resumen. El sistema de manejo de los suelos agrícolas puede modificar sustancialmente la dinámica del $\mathrm{CO}_{2}$ del suelo. Se realizó un experimento en Lanjarón (SE España) para evaluar el efecto de tres sistemas de manejo de suelos en plantaciones de almendro: 1) mínimo laboreo y cubierta de vegetación espontánea (MLVE), 2) mínimo laboreo y cubierta de tomillo (Thymbra capitata L.) (MLT), y 3) mínimo laboreo sin cubiertas vegetales (ML). Las tasas de emisión del $\mathrm{CO}_{2}$ del suelo presentaron el siguiente orden creciente $\mathrm{MLVE}>\mathrm{ML}>\mathrm{MLT}$, determinándose una alta variabilidad a corto plazo. Así mismo, se registraron mayores emisiones de $\mathrm{CO}_{2}$ durante la primavera, en contraste a las de otoño. Por otra parte, el contenido de agua en el suelo fue el factor ambiental con mayor influencia en las emisiones. En consecuencia, el empleo de técnicas de agricultura de conservación como el mínimo laboreo y cubiertas vegetales para el secuestro de $\mathrm{C}$ atmosférico son medidas sostenibles en los agroecosistemas mediterráneos de secano.

Palabras clave: Cambio climático; $\mathrm{CO}_{2}$; cubierta vegetal; mínimo laboreo; Prunus dulcis Mill.

\section{[en] Effect of different conservation agriculture techniques on soil respiration in almond orchards in the Mediterranean region (SE Spain)}

\begin{abstract}
The soil-management system in agricultural lands could be affected substantially on dynamics of $\mathrm{CO}_{2}$ fluxes from soil. An experiment was carried out in Lanjarón (SE Spain) to evaluate the effect of three soil-management systems in almond plantations: 1) minimum tillage with spontaneous vegetation strips (MTSV), 2) minimum tillage and thyme (Thymbra capitata L.) strips (MTT), and 3) minimum tillage without plant strips (MT). The $\mathrm{CO}_{2}$ emission rates from soil presented the following increasing trend MTSV $>\mathrm{MT}>\mathrm{MTT}$, showing highly variability in the short term. Also, higher $\mathrm{CO}_{2}$ soil emissions were recorded during the spring, in contrast to autumn. Moreover, the soil- water content was the most influential environmental factor. Consequently, the use of conservation agricultural techniques such as minimum tillage and plant strips to sequester atmospheric carbon are sustainable measures for Mediterranean rainfed agro-ecosystems.
\end{abstract}

Key words: Climate change; $\mathrm{CO}_{2}$; minimum tillage; plant cover; Prunus dulcis Mill.

IFAPA Centro “Camino de Purchil”. (Granada, España).

IFAPA Centro "Venta del Llano". Parque Científico y Tecnológico GEOLIT (Mengíbar, Jaén, España).

IFAPA Centro "Las Torres-Tomejil”. (Alcalá del Río, Sevilla, España).

E-mail: víctor.duran@juntadeandalucia.es 
[fr] Effet des techniques d'agriculture de conservation sur la respiration du sol dans les plantations d'amandiers de la région méditerranéenne (SE Espagne)

Résumé. Le système de gestion des sols agricoles peut modifier substantiellement la dynamique du $\mathrm{CO}_{2}$ du sol. Une expérience a été realisé à Lanjarón (SE Espagne) pour évaluer l'effet produit pour trois systèmes de gestion des sols dans des plantations d'amandiers: 1) travail réduit du sol est couvert de végétation naturelle $\left.\left(\mathrm{TR}_{\mathrm{vn}}\right), 2\right)$ travail réduit du sol et couvert de thym (Thymbra capitata $\mathrm{L}$.) (TRT) et 3) travail reduit sans couvert vegetaux (TR). Des taux d'émission du $\mathrm{CO}_{2}$ du sol ont eu l'ordre suivant: TRvn $>$ TR $>$ TRT et une importante variabilité ont été trouvés à court terme. De la même manière, des plus grands remisaient de $\mathrm{CO}_{2}$ a été trouvé en printemps, alors que des emision en automnes ont été minimums. D'autre part, le contenu d'eau dans le sol a été le principal factor environnemental ayant d'influence sur des emsions. Par conséquent, l'utilisation des techniques d'agriculture de conservation comme le travail réduit du sol et l'emploi des couverts végétaux pour la séquestration de carbone atmosferique sont des mesures durables dans des agro-systemes méditerranées non irriguées.

Mots clés: Changement climatique; $\mathrm{CO}_{2}$; couverts vegetaux; travail redduit du sol;Prunus dulcis Mill.

Cómo citar. Rodríguez Pleguezuelo, C.R., Francia Martínez, J.R., Cárceles Rodríguez, B., Gálvez Ruiz, B., García Tejero, I.F. y Durán Zuazo, V.H. (2017): Efecto de técnicas de agricultura de conservación en la respiración del suelo en plantaciones de almendro en la región mediterránea (SE España). $M+A$ Revista Electrónica de Medioambiente, 18(2), 163-176.

Sumario. 1. Introducción. 2. Metodología. 2.1. Zona de estudio y parcelas experimentales. 2.2. Emisiones de $\mathrm{CO} 2$ del suelo y secuestro de $\mathrm{C}$ por las cubiertas. 2.3. Mediciones de la humedad del suelo. 2.4. Análisis estadístico. 3. Resultados. 4. Discusión. 5. Conclusiones. 6. Referencias bibliográficas

\section{Introducción}

Según el Panel Intergubernamental sobre Cambio Climático, la agricultura es uno de los principales sectores responsables del incremento en la concentración de gases de efecto invernadero (GEI) que ha ocurrido en los últimos 30 años (IPCC 2014). La agricultura, la selvicultura y otros usos del suelo son responsables del $24 \%$ de las emisiones de GEI en 2010 (IPCC 2014).

En concreto, la actividad agraria en España genera emisiones significativas de GEI a la atmósfera. Así en 2013, el sector agrícola español emitió 40,2 Tg de CO2-eq (MAGRAMA 2015), valor que representa más del $12 \%$ de las emisiones totales del país. Algunos de los pronósticos a largo plazo para la región mediterránea establecen además como efectos del cambio climático, una disminución de la precipitación neta y un aumento en su intensidad (Christensen et al. 2007). Afectando de forma importante a la rentabilidad de las explotaciones agrarias. Valverde et al. (2015) estima que la producción del almendro (Prunus dulcis Mill.) en secano se verá reducida un $27,2 \%$ a consecuencia del cambio climático.

Por otra parte, prácticas como el laboreo intensivo han agotado el carbono (C) orgánico del suelo, ya que se caracterizan por tener un bajo retorno de material orgánico al suelo y favorecen mecanismos como la oxidación, la mineralización, la lixiviación y la erosión (Crittenden et al. 2015; Martínez et al. 2005; Peregrina 2016). 
Este contexto refleja la urgente necesidad de adoptar medidas para el secuestro de C atmosférico en estos suelos a través de la adopción de prácticas de manejo alternativas (Moreno et al. 2010). De acuerdo con Lal et al. (2011), el manejo del suelo en la agricultura es de las mejores herramientas para la mitigación del cambio climático a través de la fijación del C atmosférico.

En este sentido, González et al. (2012) concluyen que las técnicas de agricultura de conservación en España podrían fijar alrededor de $2 \mathrm{Gg}$ de $\mathrm{C}$ al año más que los sistemas de laboreo tradicional. Además, el incremento del contenido de $\mathrm{C}$ en suelos agrícolas implica una serie de beneficios para la adaptación al cambio climático, ya que mejora las propiedades físicas, químicas y biológicas del suelo (Lal et al. 2011; Söderström et al. 2014).

En concreto, la agricultura de conservación en cultivos leñosos, permite la acumulación de $\mathrm{C}$ en el suelo por diversas razones. En primer lugar, se reduce la pérdida de materia orgánica adsorbida a los sedimentos al reducir la erosión hídrica (Durán et al. 2012; Francia et al. 2006; Gómez et al. 2009,). El segundo motivo está relacionado con el incremento del contenido de materia orgánica al ser mayor la cantidad de residuos vegetales que se incorporan al suelo (Moreno et al. 2009; Nieto et al. 2012; Ramos et al. 2010). Y por último, se reduce la mineralización de la materia orgánica al ser menor la aireación del suelo por la reducción y/o supresión del laboreo (Beare et al. 1994; Doran 1980; Melero et al. 2009).

Por otra parte, los sistemas de conservación del suelo también contribuyen a la disminución de emisiones de $\mathrm{CO} 2$ asociados a las labores agrícolas, debido al menor consumo de combustible por la maquinaría (Govaerts et al. 2009).

El objetivo de este estudio fue evaluar el impacto de diferentes prácticas de la agricultura de conservación, consistente en el mínimo laboreo y cubiertas vegetales de vegetación espontánea y matorral, en plantaciones de almendro en secano, respecto a la respiración del suelo y al secuestro de carbono.

\section{Metodología}

\subsection{Zona de estudio y parcelas experimentales}

El experimento se realizó en Lanjarón (SE España). El clima de la zona es mediterráneo con una precipitación media anual de $490 \mathrm{~mm}$ y una temperatura media anual de $15^{\circ} \mathrm{C}$. Presenta una gran irregularidad interanual e intra-anual en cuanto a la precipitación. El suelo de la zona es Typic Xerorthent, según la clasificación del Soil Survey Staff (2014).

Las parcelas experimentales forman parte de una plantación de almendros (Prunus dulcis (Mill.) D.A. Webb. cv. Desmayo Largueta) de secano (latitud y longitud de $36^{\circ}$ 54' 14,66" y 03 29' 11,89", respectivamente), con árboles espaciados a 6 x $6 \mathrm{~m}$, en una ladera del $35 \%$ de pendiente y a una altitud de 580 m s.n.m. 
Se ensayaron tres tipos de manejo del suelo: 1) mínimo laboreo y cubierta vegetal de franjas de vegetación espontánea (MLVE), 2) mínimo laboreo y cubierta vegetal de franjas de tomillo (Thymbra capitata (L.) Cav) (MLT), y 3 ) mínimo laboreo sin cubiertas vegetales (ML). Cada uno de los tratamientos se ensayó con dos repeticiones. Las operaciones de mínimo laboreo en las parcelas experimentales consistieron en una labor superficial $(0,15 \mathrm{~cm})$ dos veces al año (primavera y otoño) con un arado de cincel, usando un cultivador. En los tratamientos con cubiertas, se plantaron franjas de tres metros de ancho entre las hileras de almendros, perpendiculares a la pendiente.

El conjunto de plantas espontáneas se compone fundamentalmente por Armeria sp., Avena sativa L., Diplotaxis virgata (Cav) DC, Malva parviflora L., Phagnalon rupestre L. DC, Velezia rígida L., Anagalis arvensis L., Brachipodium sp., Bromus matritensis L. Cent., Calendula arvensis L., Campanula sp., Convolvulus altheoides L., Crepis sp., Medicago sp., Papaver rhoeas L., Raspitrum rugosum L. Sisymbrium sp., Sonchus arvensis L., Trigonella monospeliaca L., etc. Las franjas en el tratamiento de MLVE se implantaron el primer año, permitiendo la colonización de las mismas por la vegetación espontánea de la zona. En los años posteriores se recolectaron semillas de los alrededores y se sembraron con las primeras lluvias de otoño, para lograr el mayor grado de cobertura posible. Además, se conservó una pequeña franja sin segar para garantizar el semillado. Las franjas donde se localizan los almendros se labraron, manteniéndolas el resto del año libres de vegetación mediante el empleo de un herbicida de traslocación.

El control de las franjas de vegetación espontánea se realizó mediante siega mecánica, en el momento en que, la competencia de éstas por el agua del suelo, podía representar una merma en la producción del cultivo. Los restos de la cubierta se dejaron sobre el suelo tras la siega.

Las franjas de matorral en el tratamiento de MLT, estaban formadas por líneas pareadas distantes $50 \mathrm{~cm}$ entre sí y separadas $75 \mathrm{~cm}$ de las siguientes. La distancia entre plantas en las hileras fue de $25 \mathrm{~cm}$. La recolección de la biomasa de matorral se realizó recolectando el $50 \%$ de la parte aérea de cada planta, a principios de mayo.

\subsection{Emisiones de $\mathrm{CO} 2$ del suelo y secuestro de $\mathrm{C}$ por las cubiertas}

Para estimar el flujo de C a través de la respiración del suelo (Rs), se empleó un analizador de gases infrarrojo no dispersivo con una cámara de respiración acoplada (Modelo EGM-4 con SRC-1, PP-Systems, Hitchin, UK) con un tiempo de medición de $124 \mathrm{~s}$, durante el cual los datos se recogen cada $4 \mathrm{~s}$ (Figura 1). El valor final es el valor promedio de todo el período. El principio en que se basa el analizador es un sistema cerrado en el que se calculan los aumentos en la concentración de $\mathrm{CO} 2$ aérea que se producen en la superficie del suelo. El EGM-4 cuenta con un sensor para la determinación simultánea de la temperatura del suelo. 
Figura 1. Equipo de medida EGM-4 para la determinación de la respiración del suelo.

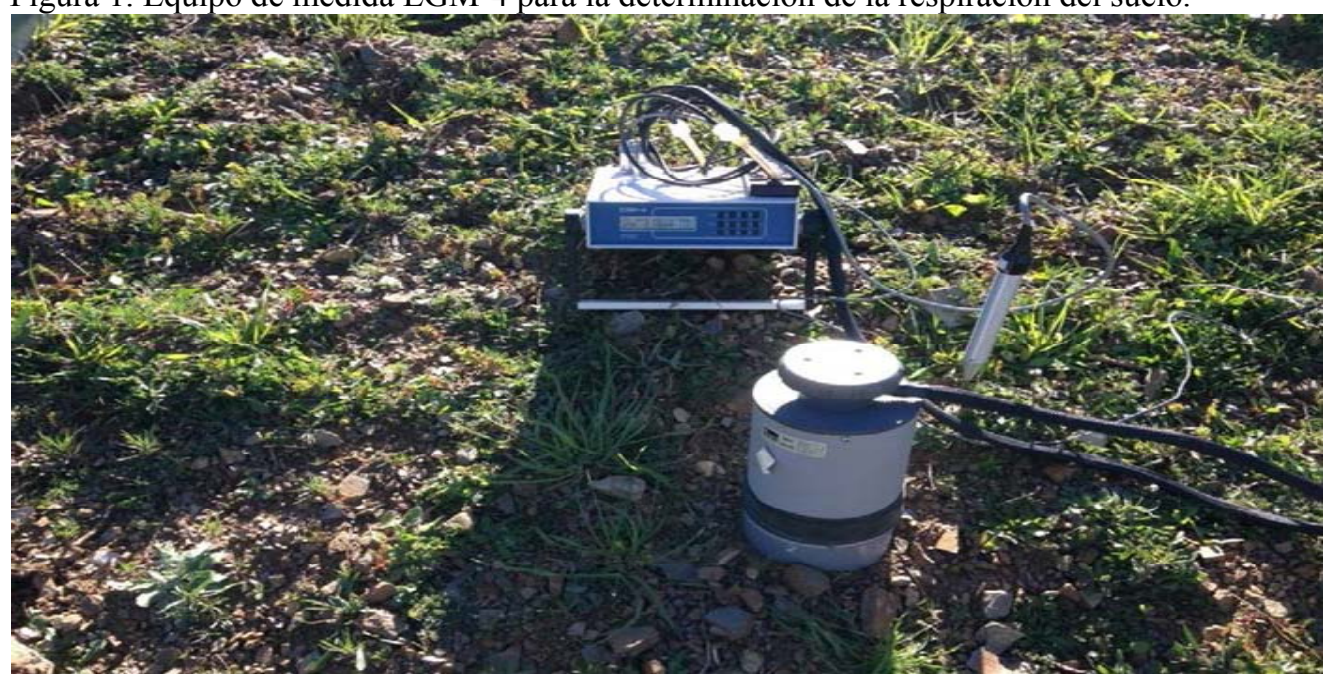

Fuente: Elaboración propia.

El flujo de salida de $\mathrm{CO} 2$ del suelo se midió mediante el ajuste de la cámara de respiración del suelo a unos anillos de cloruro de polivinilo (PVC) de $5 \mathrm{~cm}$ de profundidad y $10 \mathrm{~cm}$ de diámetro, que se colocaron en las parcelas experimentales al inicio del estudio y se mantuvieron en su lugar durante el periodo de medidas. En cada parcela se colocaron cuatro anillos de PVC, dos en las filas entre los árboles y los otros dos en la zona de árbol. Las mediciones de flujo se realizaron mensualmente desde enero de 2013 hasta abril de 2015, y siempre entre las 10:00 y las 13:00 h, ya que se ha demostrado que los valores de flujo de $\mathrm{CO} 2$ al mediodía son representativos de la media diaria de acuerdo con Álvaro et al. (2007).

Para estimar el secuestro de $\mathrm{C}$ por las cubiertas vegetales, se estimó la producción promedio anual de las cubiertas vegetales en las parcelas de MLVE y MLT, para los años 2014 y 2015 . Se determinó la materia seca, secando en estufa a $65{ }^{\circ} \mathrm{C}$, hasta pesoconstante. Posteriormente, se determinó el contenido de carbono con un analizador elemental (FISONS EA 1108 CHNSO, Carlo Erba ${ }^{\circledR}$ ).

\subsection{Mediciones de la humedad del suelo}

La humedad del suelo se midió con sondas FDR (10HS, Decagon Devices, Pullman, USA), instaladas en las parcelas experimentales. Las parcelas con cubiertas vegetales contaron con dos sondas y fueron dispuestas, uno en la zona de la cubierta y la otra en el suelo desnudo. En las parcelas con mínimo laboreo sin cubierta vegetal (ML), se instaló una sola sonda. Las sondas registran automáticamente la humedad del suelo a los $10 \mathrm{~cm}$, en intervalos de una hora, y se almacenan los datos en un datalogger (EM50, Decagon Devices). 
Para el registro de los datos micro-meteorológicos se contó con una pequeña estación meteorológica (WatchDog Data Logger Model 450, Spectrum Technologies, Aurora, USA.), instalada en las parcelas experimentales.

\subsection{Análisis estadístico}

Se realizó un análisis estadístico con el software Statistix al nivel de significación de $\mathrm{p}<0,05$. La normalidad de los datos fue analizada por medio de un test de Kolmogorov-Smirnov y la homocedasticidad con el test de Barlett. Cuando la variable estudiada incumplía ambos requisitos, y no podía ser transformada, se aplicó el test no paramétrico de Kruskal-Wallis. Así mismo se realizó un análisis de correlación.

\section{Resultados}

En la Figura 2 se muestran los datos de emisión de $\mathrm{CO} 2$ por la respiración del suelo, para cada uno de los tratamientos, a lo largo del período de estudio. El valor representado es la media de todas las medidas de respiración del suelo por tratamiento.

Figura 2. Emisiones de $\mathrm{CO} 2$ diarias por sistema de manejo de suelo durante el periodo de estudio $^{4}$.

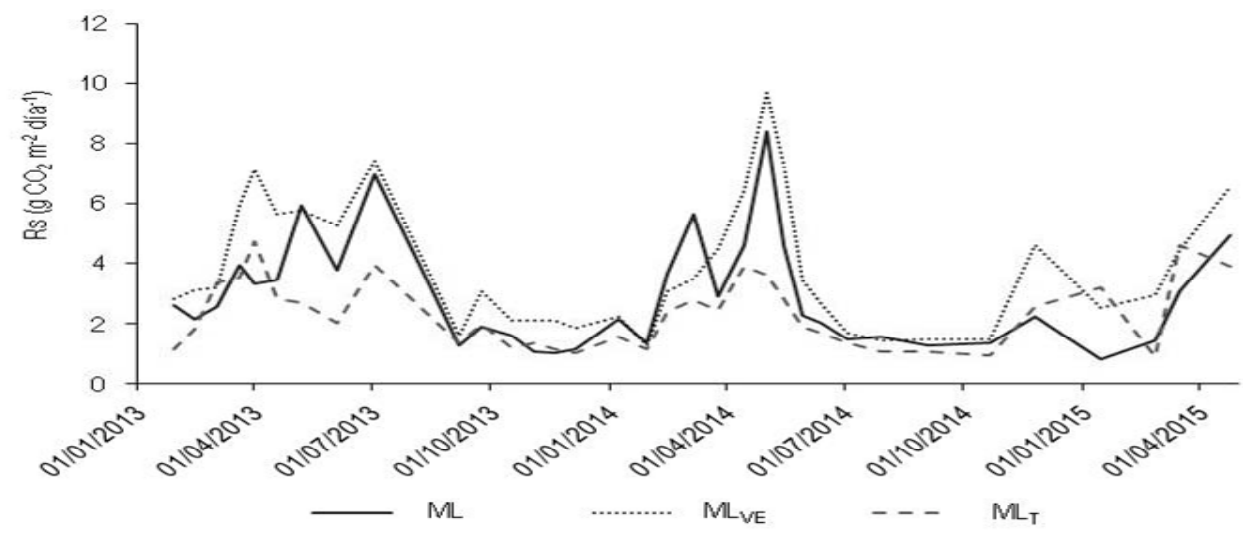

Fuente: Elaboración propia.

${ }^{4}$ ML, mínimo laboreo; MLVE, mínimo laboreo y cubierta de vegetación espontánea; MLT, mínimo laboreo y cubierta de tomillo. 
Los valores medios de la respiración del suelo por estación y manejo para el conjunto de las mediciones realizadas se presentan en el Cuadro 1. Como se observa en la misma, los mayores valores de emisión se producen durante la primavera, presentando diferencias estadísticamente significativas, especialmente con el otoño.

Cuadro 1. Respiración del suelo (RS) por estación, en función del tratamiento ${ }^{5}$.

\begin{tabular}{|c|c|c|c|}
\hline \multirow{2}{*}{ Estación } & \multicolumn{3}{|c|}{ Rs } \\
\cline { 2 - 4 } & $\mathrm{ML}$ & $\mathrm{ML}_{\mathrm{VE}}$ & $\mathrm{ML}_{\mathrm{T}}$ \\
\cline { 2 - 4 } & \multicolumn{3}{|c|}{$\left(\mathrm{g} \mathrm{CO}_{2} \mathrm{~m}^{-2} \mathrm{dia}^{-1}\right)$} \\
\hline Otoño & $1,48 \pm 0,17 \mathrm{a}$ & $2,48 \pm 0,40 \mathrm{a}$ & $1,47 \pm 0,23 \mathrm{a}$ \\
\hline Invierno & $2,55 \pm 0,43 \mathrm{ab}$ & $2,90 \pm 0,26 \mathrm{a}$ & $2,29 \pm 0,38 \mathrm{ab}$ \\
\hline Primavera & $4,18 \pm 0,50 \mathrm{~b}$ & $5,86 \pm 0,53 \mathrm{~b}$ & $3,01 \pm 0,27 \mathrm{~b}$ \\
\hline Verano & $2,51 \pm 1,12 \mathrm{ab}$ & $2,74 \pm 1,18 \mathrm{a}$ & $1,77 \pm 0,55 \mathrm{ab}$ \\
\hline
\end{tabular}

Fuente: Elaboración propia.

Cuando se estudian las emisiones en función del tratamiento con el conjunto de valores obtenidos durante el estudio, se observa que las mayores emisiones se generan en el MLVE (3,83 g CO2 m-2 día-1) y las menores en MLT (2,30 g CO2 m-2 día-1), encontrando diferencias estadísticamente significativas $(\mathrm{p}<0,05)$ entre ambos sistemas de manejo del suelo. EL ML presenta valores intermedios (2,90 g CO2 m-2 día-1) y sin diferencias significativas con los otros dos tratamientos.

Al comparar los valores de emisión medidos en la zona de la calle y los de la zona de árbol, se observa que existen diferencias estadísticamente significativas entre las dos zonas para las parcelas de MLVE y MLT, debido a la presencia de la cubierta vegetal (Cuadro 2). Sin embargo, en la parcela de ML, aunque los valores de emisión son mayores en la zona de la calle, no hay diferencias estadísticamente significativas con las mediciones realizadas en la zona del árbol.

\footnotetext{
${ }^{5}$ ML, mínimo laboreo; MLVE, mínimo laboreo y cubierta de vegetación espontánea; MLT, mínimo laboreo y cubierta de tomillo. Media \pm Error estándar; Valores con diferentes letras en la misma columna presentan diferencias estadísticamente significativas al nivel $p<$ 0,05 (Kruskal-Wallis).
} 
Cuadro 2. Respiración del suelo (RS) en la zona de calle y en la de árbol ${ }^{6}$.

\begin{tabular}{|c|c|c|c|}
\hline \multirow{2}{*}{ Zona } & \multicolumn{3}{|c|}{ Rs } \\
\cline { 2 - 4 } & \multicolumn{3}{|c|}{$\left(\mathrm{g} \mathrm{CO}_{2} \mathrm{~m}^{-2} \mathrm{dí}^{-1}\right)$} \\
\cline { 2 - 4 } & \multicolumn{3}{|c|}{ ML $_{\mathrm{T}}$} \\
\hline Calle & $3,14 \pm 0,31 \mathrm{a}$ & $4,37 \pm 0,35 \mathrm{~b}$ & $2,70 \pm 0,22 \mathrm{~b}$ \\
\hline Árbol & $2,66 \pm 0,22 \mathrm{a}$ & $3,30 \pm 0,33 \mathrm{a}$ & $1,89 \pm 0,17 \mathrm{a}$ \\
\hline
\end{tabular}

Fuente: Elaboración propia.

Se ha determinado que existe relación significativa entre el conjunto de las medidas de respiración del suelo y la humedad del suelo a $10 \mathrm{~cm}$ de profundidad, pero no con la temperatura del suelo (Cuadro 3). Encontrando correlación entre respiración del suelo y la temperatura del suelo, únicamente durante el invierno ( $\mathrm{R} 2=0,5116)$, donde la temperatura presumiblemente limita la actividad microbiológica del suelo.

Cuadro 3. Coeficientes de correlación lineal entre variables ${ }^{7}$.

\begin{tabular}{|l|l|l|l|}
\hline & Rs & T & H \\
\hline Rs & 1 & & \\
\hline$T$ & $0,0334 \mathrm{~ns}$ & 1 & \\
\hline$H$ & $0,2642 *$ & $-0,5366 *$ & 1 \\
\hline
\end{tabular}

Fuente: Elaboración propia.

En el Cuadro 4 se presentan los valores producción y de secuestro de C de la cubierta vegetal. En este sentido, la producción de biomasa de tomillo fue mayor para el 2015, debido al mayor desarrollo del matorral a medida que aumenta el período de establecimiento en el terreno y la respuesta a la siega. La producción de la cubierta de vegetación espontánea fue más dependiente de la pluviometría de cada campaña. En general, para MLVE y MLT fue de 0,45 y 0,52 t ha-1 año-1, respectivamente.

${ }^{6}$ ML, mínimo laboreo; MLVE, mínimo laboreo y cubierta de vegetación espontánea; MLT, mínimo laboreo y cubierta de tomillo. Media \pm Error estándar; Valores con diferentes letras en la misma columna presentan diferencias estadísticamente significativas al nivel $\mathrm{p}<0,05$ (Kruskal-Wallis).

$\mathrm{RS}$, respiración del suelo; $\mathrm{T}$, temperatura del suelo; $\mathrm{H}$, humedad del suelo; ns, no significativo; *, estadísticamente significativo $(\mathrm{p}<0,01)$ 
Cuadro 4. Producción y secuestro de C cubiertas vegetales ${ }^{8}$.

\begin{tabular}{|l|ll|}
\hline Cubierta/año & Materia seca & Secuestro C \\
\hline Thymbra capitata & & $\left(\mathrm{t} \mathrm{ha}^{-1} \mathrm{año}^{-1}\right)$ \\
\hline 2014 & $0,71 \pm 0,06$ & $0,37 \pm 0,03$ \\
\hline 2015 & $1,27 \pm 0,38$ & $0,66 \pm 0,20$ \\
\hline Vegetación espontánea & & \\
\hline 2014 & $0,92 \pm 0,14$ & $0,43 \pm 0,07$ \\
\hline 2015 & $1,00 \pm 0,10$ & $0,47 \pm 0,05$ \\
\hline
\end{tabular}

Fuente: Elaboración propia.

\section{Discusión}

En los tres tratamientos de suelo estudiados, los valores de respiración cambiaron de forma notable a lo largo del año, en función de la temperatura y la disponibilidad de agua, como se ha observado previamente por otros estudios en ambientes mediterráneos (Almagro et al. 2009; Álvaro et al. 2008).

Es lógico que los valores más altos de respiración del suelo se hayan registrado en primavera, dado que la actividad biológica del suelo es máxima durante esta estación (Guidolotti et al. 2013; Xu et al. 2004), debido a las temperaturas templadas y a la disponibilidad de agua por las lluvias.

El hecho de que para el MLVE la respiración del suelo presente diferencias significativas entre el resto de estaciones y no sólo con el otoño, como ocurre para los otros dos tratamientos, puede deberse al aumento de $\mathrm{C}$ por la presencia de los restos de la cubierta vegetal segada sobre el suelo.

En general, los valores de Rs registrados en nuestro estudio de 13,99, 10,59 y 8,38 t CO2 ha-1 año-1, para MLVE, ML y MLT, respectivamente, fueron superiores a los obtenidos por otros autores para cultivos leñosos en secano en condiciones mediterráneas. La abundante presencia de distintas plantas anuales y espontáneas en MLVE, y su gran facilidad para iniciar el proceso de degradación de los restos, conjuntamente con las grietas que se producen en el suelo debido a la descomposición de las raíces, pueden provocar una mayor salida de los gases del suelo hacia la atmósfera. Este hecho contrasta con el tratamiento MLT, en el cual debido a su naturaleza de matorral perenne, está menos expuesto a los procesos de degradación a corto plazo. Así mismo, en el tratamiento MLVE se propician las condiciones favorables para el establecimiento y desarrollo de la artropodofauna, que hallan un lugar de refugio y alimentoz1. Equilibrio natural que es perturbado por el laboreo

\footnotetext{
${ }^{8}$ Media \pm Desviación estándar
} 
convencional en cultivos leñosos de secano en zonas mediterráneas según Sánchez et al. (2015).

Respecto al sistema de ML sin cubiertas, la situación fue intermedia en términos de volumen de flujo de $\mathrm{CO} 2$ del suelo. En este sentido, existe una cierta evidencia en cuanto al incremento de los flujos de $\mathrm{CO} 2$ del suelo por efecto de las cubiertas vegetales (Sanz et al. 2014), siendo su papel como herramienta de secuestro de C en los sistemas agrícolas incuestionable. Almagro et al. (2013) en almendros ecológicos en secano en la región de Murcia registran valores de Rs de 4,95 y 5,18 t CO2 ha-1 año-1 en laboreo reducido y laboreo reducido con enmienda verde, respectivamente. Para viñedo en California, Carlisle et al. (2006) determina flujos de C por Rs de 7,02 t ha-1 año-1, labrando dos veces al año y Steenwerth et al. (2010) valores medios de 9,07, 9,57 y 9,70 t CO2 ha-1 año-1 con cubierta vegetal de cebada segada, cubierta vegetal de cebada segada e incorporada al suelo mediante laboreo y cubierta de vegetación espontánea segada e incorporada al suelo mediante laboreo, respectivamente. Así mismo, en un olivar de secano arado regularmente, Almagro et al. (2010) registran tasas de emisión de 4,27 t CO2 ha-1 año-1.

Esta variabilidad puede deberse al sistema de medición empleado, ya que a diferencia de otros estudios (Abdalla et al. 2014; Carlisle et al. 2006; Rey et al. 2011) no se eliminó la vegetación, los restos vegetales y/o insectos presentes en el suelo, antes de realizar la medida. De forma que se han podido sobreestimar los valores de emisión, al no discriminarse entre la respiración del suelo y la posible contribución de la cubierta vegetal o los restos orgánicos del suelo.

Las diferencias en los valores obtenidos no sólo deben atribuirse al sistema de medida de la Rs, ya que la magnitud de la respuesta de los sistemas de agricultura de conservación en el secuestro de $\mathrm{C}$ y la reducción de las emisiones, variaba considerablemente dependiendo de la profundidad de las labores realizadas en el suelo y las condiciones edáficas y climáticas de la zona (Carbonell et al. 2011), lo que hace a veces difícil de comparar los valores obtenidos por diferentes estudios.

Por otra parte, López et al. (2014) han mostrado como los flujos de CO2 son bastante inestables en los experimentos a corto plazo, mientras que tienden a ser más constantes a largo plazo.

Además del sistema de medida empleado, como ya se explicó anteriormente, los mayores valores de Rs registrados en la zona de la calle, incluso para la parcela de ML, pueden deberse a una mayor densidad de raíces superficiales y de biomasa microbiana en la zona de la calle que en la zona del árbol, ya que ésta última se ve afectada por el paso de la maquinaría para realizar las labores y por la aplicación de herbicidas.

El hecho de que para el conjunto de los datos sólo se haya encontrado correlación para la Rs con la humedad del suelo, y no con la temperatura, coincide con diversos estudios (Almagro et al. 2009; Conant et al. 2000; Liu et al. 2009; Maestre y Cortina 2003) que establecen que en los ecosistemas semiáridos, el contenido de agua del suelo es el limitante principal de la actividad biológica y la Rs. El clima seco y cálido del sudeste español hace que la dinámica estacional de la Rs esté controlada 
principalmente por la disponibilidad de agua y sólo ocasionalmente, como ocurre en el invierno, por la temperatura (Domingo et al. 2011). En este sentido, Rey et al (2002) encontraron que la temperatura del suelo juega un papel importante en el control de Rs cuando el contenido volumétrico de agua aumenta por encima de un valor umbral del $20 \%$, siendo la misma controlada por la humedad del suelo por debajo de ese valor.

Los valores de secuestro de $\mathrm{C}$ por cubiertas vegetales son algo superiores a los que estima Aguilera et al. (2013) de 0,27 t C ha-1 año-1 para suelos con cultivos leñosos, y bastante inferiores de los obtenidos por González-Sánchez et al. (2012) en un estudio realizado en España, donde las cubiertas vegetales se asocian con una media de secuestro de C de 1,59 t C ha-1 año-1.

\section{Conclusiones}

La aplicación de las técnicas de agricultura de conservación, como el mínimo laboreo y cubiertas vegetales de tomillo en cultivos leñosos de secano permitieron reducir las emisiones de $\mathrm{CO} 2$ a la atmósfera, al mismo tiempo se evidencia una alta tasa de emisiones por la cubierta vegetal de plantas espontáneas.

Se determina que tanto las emisiones como la fijación de $\mathrm{C}$ no son estables en el corto plazo, por lo que se requiere de estudios de larga duración para determinar la influencia de las diferentes prácticas agrícolas. Por otra parte, en las condiciones climáticas del sudeste español, el contenido de agua en el suelo fue el factor ambiental con mayor influencia sobre la respiración del suelo. Así mismo, el secuestro de $\mathrm{C}$ por medio de las cubiertas fue particularmente relevante, siendo esto muy importante en los agro-ecosistemas mediterráneos, donde los suelos son usualmente muy sensibles y vulnerables a los efectos de la desertificación.

En consecuencia, se requiere una alternativa a las prácticas convencionales de laboreo que favorecen las emisiones de $\mathrm{CO} 2$ y el deterioro de los horizontes superficiales del suelo, impacto negativo que puede ser reducido en cierta medida con la aplicación de mínimo laboreo en un contexto de agricultura de conservación.

\section{Referencias bibliográficas}

Abdalla M, Hastings A, Helmy M, Prescher A, Osborne B, Lanigan G, Forristal D, Killi D, Maratha P, Williams M, Rueangritsarakul K, Smith P, Nolan P, Jones MB (2014): Assessing the combined use of reduced tillage and cover crops for mitigating greenhouse gas emissions from arable ecosystem. Geoderma 223-225: 9-20.

Aguilera E, Lassaletta L, Gattinger A, Gimeno BS (2013): Managing soil carbon for climate change mitigation and adaptation in Mediterranean cropping systems: A meta-analysis. Agriculture, Ecosystems and Environment 168: 25-36. 
Almagro M, López J, Querejeta JI, Martínez MM (2009): Temperature dependence of soil $\mathrm{CO} 2$ efflux is strongly modulated by seasonal patterns of moisture availability in a Mediterranean ecosystem. Soil Biology and Biochemistry 41: 594-605.

Almagro M, López J, Boix-Fayos, Albaladejo J, Martínez MM (2010): Belowground carbon allocation patterns in a dry Mediterranean ecosystems: A comparison two models. Soil Biology \& Biochemistry 42: 1549-1557.

Almagro M, de Vente J, Boix-Fayos C, García FN, Melgares de Aguilar J, González D, SoléBenet A, Martínez MM (2013): Sustainable land management practices as providers of several ecosystem services under rainfed Mediterranean agroecosystems. Mitigation and Adaptation Strategies for Global Change 1-15.

Álvaro FJ, Cantero MC, López MV, Arrúe JL (2007): Soil carbon dioxide fluxes following tillage in semiarid Mediterranean agroecosystems. Soil \& Tillage Research 96: 331-341.

Álvaro FJ, López MV, Arrúe JL, Cantero MC (2008): Management effects on soil carbon dioxide fluxes under semiarid Mediterranean conditions. Soil Science Society of America Journal 72: 194-200.

Beare MH, Hendrix PF, Cabrera ML, Coleman DC (1994): Aggregate-protected and unprotected organic matter pools in conventional-and no-tillage soils. Soil Science Society of America Journal 58(3): 787-795.

Carbonell BR, González SEJ, Veróz GO, Ordóñez FR (2011): Soil management systems and short term $\mathrm{CO} 2$ emissions in a clayey soil in southern Spain. Science of the Total Environment 409: 2929-2935.

Carlisle EA, Steenwerth KL, Smart DR (2006): Effects of land use on soil respiration: conversion of oak woodlands to vineyards. Journal of Environmental Quality 35: 13961404.

Christensen, JH, Hewitson B, Busuioc A, Chen A, Gao X, Held I, Jones R, Kolli RK, Kwon WT, Laprise R, Rueda VM, Mearns L, Menéndez CG, Räisänen J, Rinke A, Whetton ASP (2007): Regional Climate Projections. En: Climate Change 2007: The Physical Science Basis. Contribution of Working Group I to the Fourth Assessment Report of the Intergovernmental Panel on Climate Change [Solomon S, Qin D, Manning M, Chen Z, Marquis M, Averyt KB, Tignor M and Miller HL (eds.)]. Cambridge University Press, Cambridge, pp: 847-943.

Crittenden SJ, Poot N, Heinen M, van Balen DJM, Pulleman MM. 2015. Soil physical quality in contrassting tillage systems in organic and conventional farming. Soil and Tillage Research 154: 136-144.

Conant SG, Klopatek JM, Klopatek CC (2000): Environmental factors controlling soil respiration in three semiarid ecosystems. Soil Science Society of American Journal 64: 383-390.

Domingo F, Serrano OP, Were A, Villagarcía L, García M, Ramírez DA, Kowalski AS, Moro MJ, Rey A, Oyonarte C (2011): Carbon and water exchange in semiarid in SE Spain. Journal of Arid Environments 75: 1271-1278.

Doran JW (1980): Soil microbial and biochemical changes associated with reduced tillage. Soil Science Society of America Journal 44(4): 765-771. 
Durán ZVH, Francia JR, García TIF, Arroyo L, Martínez RA (2012): Mitigación de la erosión de suelos en plantaciones de almendro por cubiertas vegetales: implicación para la agricultura sostenible de montaña (SE España). Comunicata Scientiae 3(2): 123-129.

Francia MJR, Durán ZVH, Martínez AR (2006): Environmental impact from mountainous olive orchards under different soil-management systems (SE Spain). Science of the Total Environment 358: 46-60.

Gómez JA, Guzmán MG, Giráldez JV, Fereres E (2009): The influence of cover crops and tillage on water and sediment yield, and on nutrient and organic matter losses in an olive orchard on a sandy loam soil. Soil \& Tillage Research 106: 137-144.

González SEJ, Ordóñez FR, Carbonell BR, Veroz GO, Gil RJA (2012): Review. Metaanalysis on atmospheric carbon capture in Spain through the use of conservation agriculture. Soil \& Tillage Research 122: 52-60.

Govaerts B, Verhulst N, Castellanos NA, Sayre KD, Dixon J, Dendooven L. 2009. Conservation agriculture and soil carbon sequestration: between myth and farmer reality. Critical Reviews in Plant Sciences 28(3): 97-122.

Guidolotti G, Rey A, D’Andrea E, Matteucci G, De Angelis P (2013): Effect of environmental variables and stand structure on ecosystem respiration components in a Mediterranean beech forest. Tree Physiology 33(9): 960-972.

IPCC. Intergovernmental Panel on Climate Change. 2014. Summary for policy makers.

Working Group III. Assessment report 5.

http://www.ipcc.ch/pdf/assessment-report/ar5/wg3/ipcc_wg3_ar5_summary-forpolicymakers.pdf. Fecha de consulta 17 de febrero de 2017.

Lal R, Delgado JA, Groffman PM, Millar N, Dell C, Rotz A (2011): Management to mitigate and adapt to climate change. Journal of Soil and Water Conservation 66 (4): 276-285.

Liu W, Zhang Z, Wan S (2009): Predominant role of water in regulating soil and microbial respiration and their responses to climate change in a semiarid grassland. Global Change Biology 15: 184-195.

López GR, Madejón E, Moreno F, Murillo JM (2014): Conservation tillage influence on carbon dynamics under Mediterranean conditions. Pedosphere 24 (1): 65-75.

Maestre FT, Cortina J (2003): Small-scale spatial variation in soil CO2 efflux in a Mediterranean semiarid steppe. Applied Soil Ecology 23: 199-209.

MAGRAMA (2015); Inventario de emisiones de gases de efecto invernadero de España. Años 1990-2013. Ministerio de Agricultura, Alimentación y Medio Ambiente, Madrid.

Martínez RA, Durán ZVH, Francia MJR (2005): Soil erosion and runoff response to plantcover strips on semi-arid slopes (SE Spain). Land Degradation \& Development 16: 1-11.

Melero S, López GR, Madejón E, Murillo JM, Vanderlinden K, Ordóñez R, Moreno F (2009): Long-term effect of conservation tillage on organic fractions in two soils in southwest of Spain. Agriculture, Ecosystem and Environment 133: 68-74.

Moreno M, García S, Cañizares R, Castro J, Benítez E (2009): Rainfed olive farming in southeastern Spain: long-term effect of soil management on biological indicators of soil quality. Agricultural Ecosystem and Environment 131: 333-339.

Moreno F, Arrúe JL, Cantero MC, López MV, Murillo JM, Sombrero A, López GR, Madejón E, Moret D, Álvaro FJ (2010): Conservation agriculture under Mediterranean conditions in 
Spain. En: Lichtfouse E (ed) Biodiversity, biofuels, agroforestry and conservation agriculture. Sustainable agriculture reviews, vol. 5, pp: 175-193. Springer.

Nieto OM, Castro J, Fernández OE (2013): Conventional tillage versus cover crops in relation to carbon fixation in Mediterranean olive cultivation. Plant Soil 365 (1): 321-335.

Peregrina F (2016): Surface soil properties influence carbon oxide pulses after precipitation events in a semiarid vineyard under conventional tillage and cover crops. Pedosphere 26: 499-509.

Ramos ME, Benítez E, García PA, Robles AB (2010): Cover crops under different managements vs. frequent tillage in almond orchards in semiarid conditions: Effects on soil quality. Applied Soil Ecology 44: 6-14.

Rey A, Pegoraro E, Tedeschi V, De Parri I, Jarvis PG, Valentini R (2002): Annual variation in soil respiration and its components in a coppice oak forest in Central Italy. Global Change Biology 8: 851-866.

Rey A, Pegoraro E, Oyonarte C, Were A, Escribano P, Raimundo J (2011): Impact of land degradation on soil respiration in a steppe (Stipa tenacissima L.) semi-arid ecosystem in the SE of Spain. Soil Biology \& Biochemestry 43: 393-403.

Sanz CA, García MC, Quemada M, Gabriel JL, Almendros P, Vallejo A (2014): Do cover crops enhance $\mathrm{N} 2 \mathrm{O}, \mathrm{CO} 2$ or $\mathrm{CH} 4$ emissions from soil in Mediterranean arable systems? Science of the Total Enviroment 1: 164-174.

Sánchez MS, Castro J, Alonso PE, Alonso PJL, García BJM, Talavera RFM, Durán ZVH (2011): Tillage and herbicide decrease soil biodiversity in olive orchards. Agronomy for Sustainable Development 35, 691-700.

Söderström B, Hedlund K, Jackson LE, Kätterer T, Lugato E, Thomsen IK, Bracht JH (2014) What are the effects of agricultural management on soil organic carbon (SOC) stocks? Environmental Evidence, 3:2

Soil Survey Staff (2014); Keys to Soil Taxonomy, 12th ed. USDA-Natural Resources Conservation Service, Washington, DC. 362P.

Steenwerth KL, Pierce DL, Carlisle EA, Spencer RGM, Smart DR (2010): A vineyard agroecosystem: Disturbance and precipitation affect soil respiration under Mediterranean conditions. Soil \& Water Management \& Conservation 74(1): 231- 238.

Valverde P, de Carvalho M, Serralheiro R, Maia R, Ramos V, Oliveira B (2015): Climate change impacts on rainfed agriculture in the Guadiana river basin (Portugal). Agricultural Water Management 150: 35-45.

$\mathrm{Xu}$ L, Baldocchi DD, Tang J (2004): How soil moisture, rain pulses, and growth alter the response of ecosystem respiration to temperature. Global Biogeochemical Cycles 18(4). 